\title{
Improved Structural and Optical Properties Of C(Carbon)-Supported Zno Nanorod Arrays
}

\author{
Yoon $\mathrm{IT}^{1 *}$ and $\mathrm{Li} \mathrm{MK}^{2}$ \\ ${ }^{1}$ Quantum Functional Semiconductor Research Center, Dongguk University, Seoul, 100-715, \\ Republic of Korea \\ ${ }^{2}$ School of Materials Science and Engineering, Hubei University, Wuhan 430062, PR China
}

ISSN: 2578-0255

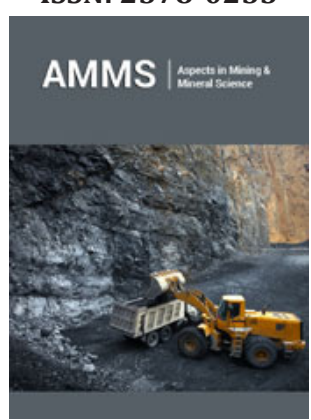

*Corresponding author: Yoon IT, Quantum Functional Semiconductor Research Center, Dongguk University, Seoul, 100715, Republic of Korea

Submission: 海 September 03, 2019

Published: 制September 10, 2019

Volume 3 - Issue 3

How to cite this article: Yoon IT, Li MK. Improved Structural and Optical Properties Of C(Carbon)-Supported Zno Nanorod Arrays. Aspects Min Miner Sci.3(3). AMMS.000567.2019.

DOI: 10.31031/AMMS.2019.03.000567

Copyright@ Yoon IT, This article is distributed under the terms of the Creative Commons Attribution 4.0 International License, which permits unrestricted use and redistribution provided that the original author and source are credited.

\begin{abstract}
We have fabricated as-grown $\mathrm{ZnO}$ nanorods (NRs) and C(carbon)-supported NRs arrays on semi-insulating (100)-oriented Si substrates. Field emission scanning electron microscopy, X-ray diffraction, revealed that the as-grown $\mathrm{ZnO}$ NRs and carbon-assisted ZnO NRs were single crystals with a hexagonal wurtzite structure, and grew with a c-axis orientation perpendicular to the Si substrate. These measurements show that the carbon-supported ZnO NRs were better synthesized vertically on a Si substrate compared to the as-grown ZnO NRs. Superconducting Quantum Interference Device showed that defect concentration of the carbon-assisted ZnO NRs was remarkably reduced compared to the as-grown ZnO NRs. The reduced defect concentration of the carbon-supported $\mathrm{ZnO}$ demonstrates the possible improvement in the performance of photovoltaic nanodevices based on $\mathrm{ZnO}$ like materials.
\end{abstract}

Keywords: ZnO nanorods; Single crystal; Vapor phase transport; Magnetization; Point defects

Abbreviations: NRs: Nanorods; LEDs: Light-Emitting Diodes; VPT: Vapor-Phase Transport; SEM: Scanning Tunneling Microscopy; SQ1UID: Superconducting Quantum Interference Device; XRD: X-Ray Diffraction

\section{Introduction}

Single-crystalline semiconducting nanostructures such as nanorods (NRs) and nanotubes show interesting physical properties and are promising the next generation of electronic and photonic devices such as light-emitting diodes (LEDs), nanowire laser and photodetectors [1-3]. Functional devices such as vertical nanowire field effect transistors, piezoelectric nanogenerators, biosensors and novel photoconductors have been demonstrated using $\mathrm{ZnO}$ nanostructures [4-7]. Meanwhile, oxygen plasma-treated sample of ZnO NRs showed the enhanced piezoelectric potential due to the decreased defects levels in the $\mathrm{ZnO}$ and also improve mechanical properties of ZnO NRs compared to the sample not treated with oxygen plasma [8]. In the present work, we investigate the structural and magnetic properties of the carbon-supported ZnO NR array compared to the sample not supported with carbon.

\section{Experimental}

As stated in previous works Yoon et al. [9] by the authors of this contribution $[9,10]$ : "we grew ZnO NRs from the semi-insulating (100)-oriented Si substrates by vapor-phase transport (VPT) technique. And then, the carbon layer with the thickness of around 9nm to assist the nucleation of vertically aligned single crystalline ZnO NRs was deposited using low pressure chemical vapor deposition method on Si substrate before the ZnO NRs growth using VPT method. The major purpose of a carbon-supported process is that it avoids undesired contamination from foreign metal atoms used as catalysts and not only to facilitate the nucleation of $\mathrm{ZnO}$ nuclei but also to decrease the lattice mismatch between the Si substrate and the $\mathrm{ZnO}$ NRs. The carbon-supported process also avoids undesired $\mathrm{SiO}_{2}$ layer on $\mathrm{Si}$ substrate to limit optoelectronic application such as field emission display, et al. The carbonsupported ZnO NRs with high quality could be grown vertically, densely oriented with respect to the surface as hexagonal pillars with a flat facet surface."

\section{Results and Discussion}

Figure 1(a) \& (b) show low- magnification scanning tunneling microscopy (SEM) image of the as-grown ZnO NRs arrays and the carbon-supported ZnO NRs arrays on semi-insulating 
(100)-oriented Si substrates. The average length of the as-grown ZnO NRs is $\sim 25 \mu \mathrm{m}$. The NR surface density was $1 \times 10^{9} / \mathrm{cm}^{2}$. The carbon-supported $\mathrm{ZnO}$ NRs have regular hexagonal shape and flat ends which vertically well aligned along the c-axis as shown in low-magnification SEM image of Figure 1(b). Figure 2(a) \& (b) show X-ray diffraction (XRD) spectra of the as-grown ZnO NRs and carbon-supported ZnO NRs on Si substrate. In Figure 2(a), XRD spectrum of the as-grown ZnO NRs shows peaks of (002), (101), (102), (103), (112), (201), and (004) indicating that the ZnO NRs have a wurtzite structure. The XRD pattern indicates that the growth direction of ZnO NRs is [0001]. No characteristic peaks from other crystalline forms were detected in the XRD pattern. Therefore, lowmagnification SEM image and XRD spectra of the as-grown ZnO NRs on Si substrate support that the grown NRs are single-crystalline NRs. Meanwhile, XRD spectrum of the carbon-supported ZnO NRs show relatively dominant (002) and (004) peaks imply that most of the carbon-supported ZnO NRs are aligned toward (002) and (004) directions compared to the as-grown ZnO NRs. Moreover, the intensity of XRD spectrum of the carbon-supported ZnO NRs was increased much more than that of the as-grown ZnO NRs, indicating higher crystal quality of the carbon-supported ZnO NRs.
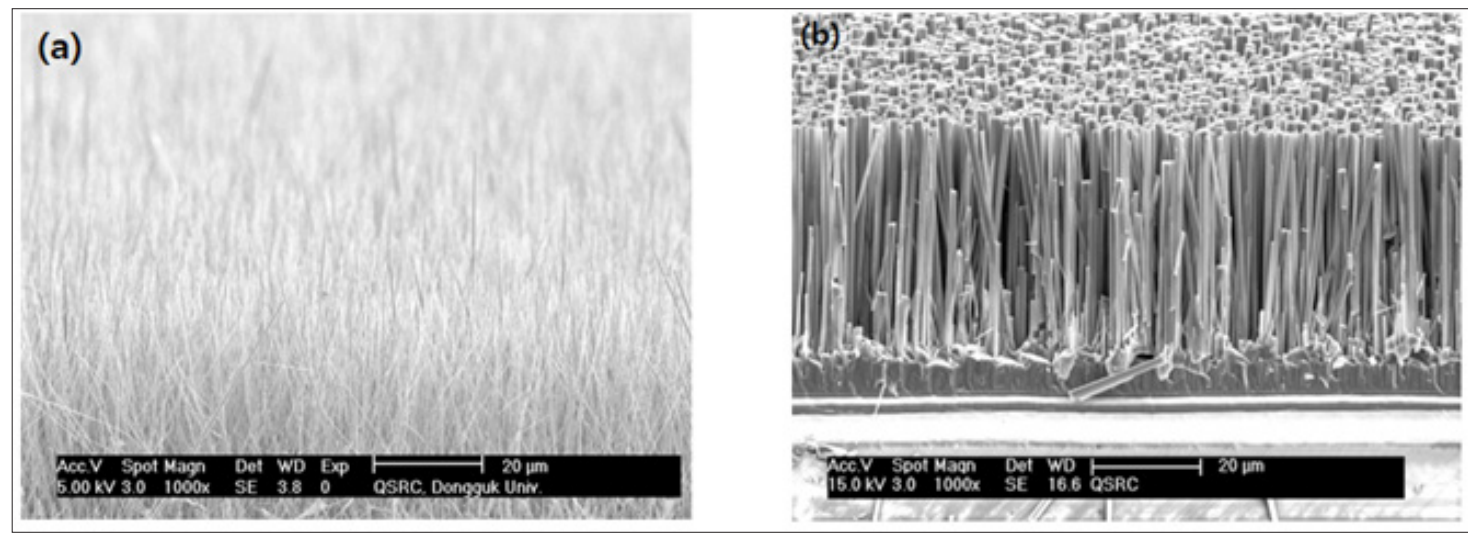

Figure 1: (a) Low magnification SEM image of the as-grown $\mathrm{ZnO}$ nanorods and (b) carbon-supported $\mathrm{ZnO}$ nanorods on Si substrate with scale bar of $20 \mu \mathrm{m}$.
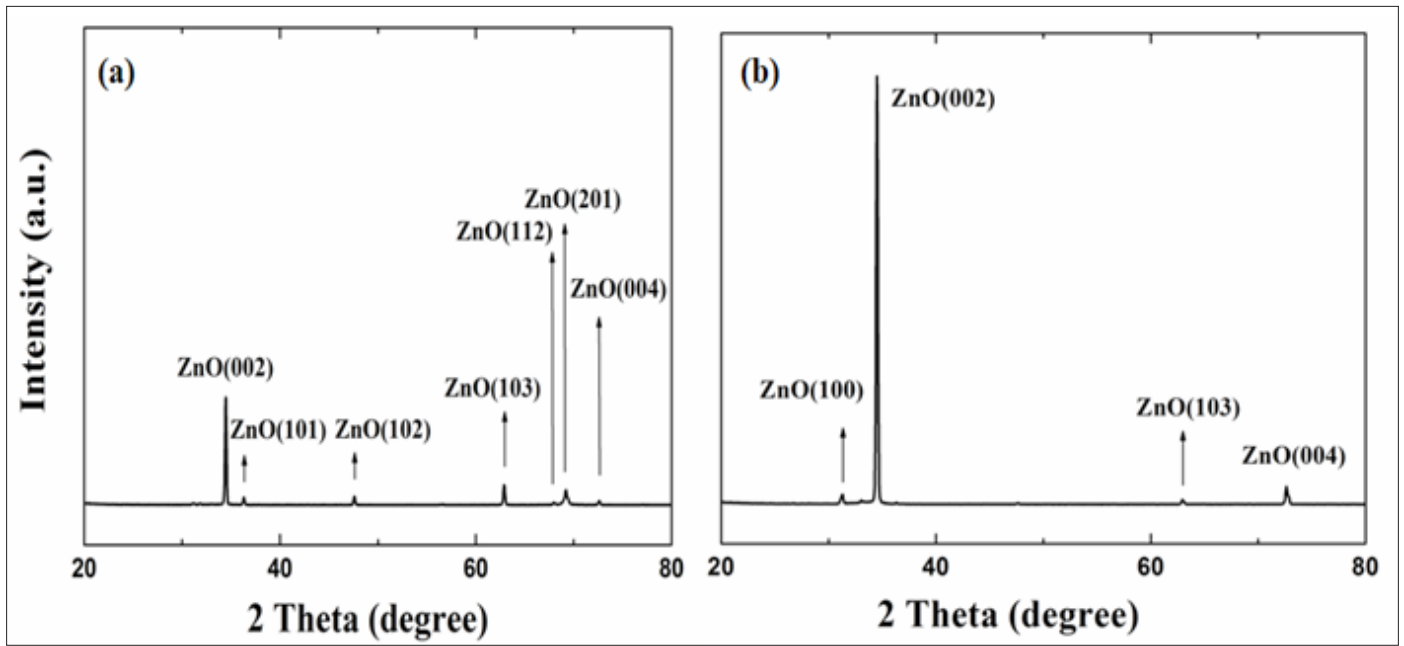

Figure 2: (a) XRD spectra of the as-grown $\mathrm{ZnO}$ nanorods and (b) carbon-supported $\mathrm{ZnO}$ nanorods on Si substrate.

Superconducting Quantum Interference Device (SQ1UID) measurement for the as grown $\mathrm{ZnO}$ NRs and carbon-supported $\mathrm{ZnO}$ NRs was carried out at 5 and $300 \mathrm{~K}$, and the results are shown in Figure $3 \& 4$. The SQUID measurement of the magnetization reveals an open hysteric curve for the as grown $\mathrm{ZnO} \mathrm{NRs}$ and carbon supported ZnO NRs, indicating that the system is ferromagnetically ordered. The carbon-supported ZnO NRs sample and as-grown sample exhibit ferromagnetic at room temperature as shown in Figure 3. Therefore, it can be seen that the ferromagnetic transition temperature of the carbon-supported ZnO NRs sample and asgrown sample is above room temperature. It is well known that the room temperature ferromagnetism is expected to be due to vacancy mediated exchange interactions between the unpaired electron spins in undoped ZnO NRs array [11]. The carbon-supported ZnO NRs sample shows very weak ferromagnetic contribution compared to the as-grown sample. The saturation magnetization of the carbonsupported ZnO NRs was decreased compared to the as grown $\mathrm{ZnO}$ NRs. SQUID measurements showed that defect concentration of the carbon-supported ZnO NRs was remarkably reduced compared to the as-grown ZnO NRs. The reduced defect concentration of the carbon-supported $\mathrm{ZnO}$ demonstrates the possible improvement in the performance of photovoltaic nanodevices based on $\mathrm{ZnO}$ like materials. 


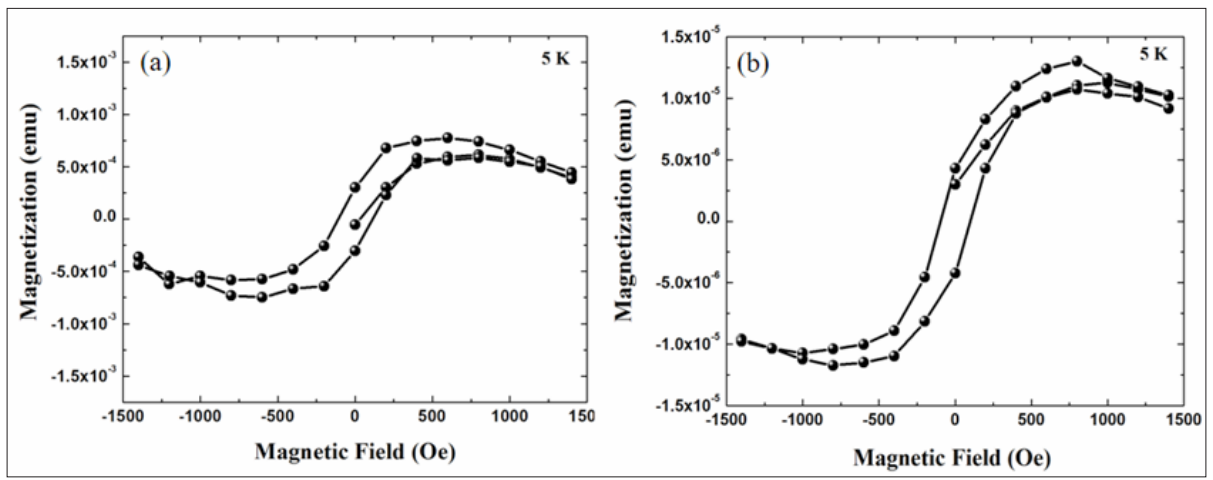

Figure 3: Ferromagnetic hysteresis loop of the (a) as-grown $\mathrm{ZnO}$ nanorods and (b) carbon-supported $\mathrm{ZnO}$ nanorods measured by SQUID magnetometer at $5 \mathrm{~K}$.
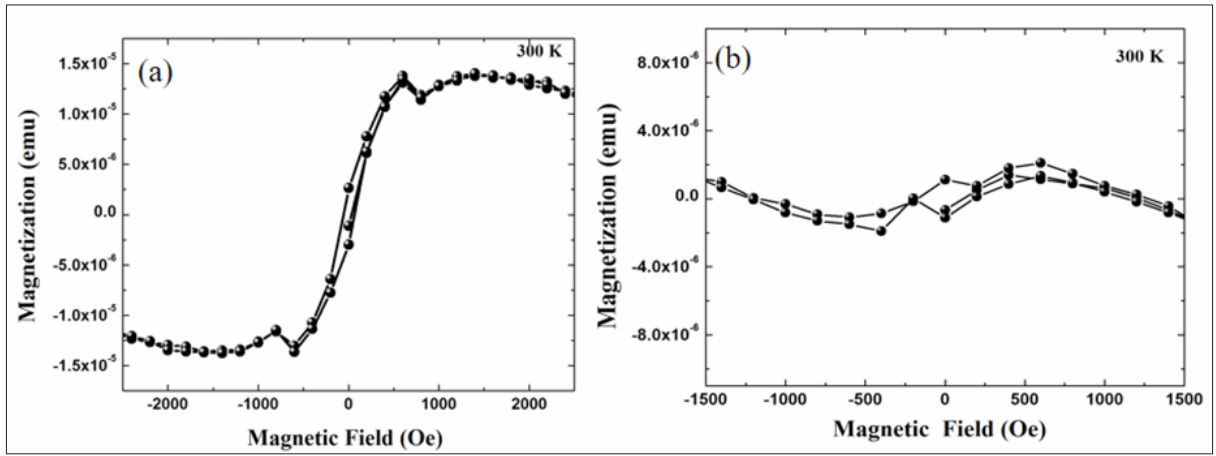

Figure 4: Ferromagnetic hysteresis loop of the (a) as-grown $\mathrm{ZnO}$ nanorods and (b) carbon-supported $\mathrm{ZnO}$ nanorods measured by SQUID magnetometer at 300K.

\section{Conclusion}

It can be concluded that the carbon-supported $\mathrm{ZnO}$ NRs were successfully synthesized on ZnO single crystal NRs with higher crystal quality compared to the as-grown ZnO NRs.

\section{Acknowledgment}

This research was supported by Basic Science Research Program through the National Research Foundation of Korea (NRF) grant funded by the Ministry of Education, Science and Technology(MEST) (NRF-2016R1D1A1B03930992).

\section{References}

1. Lu J, Shi Z, Wang Y, Lin Y,Zhu Q etal. (2016) Plasmon-enhanced electrically light-emitting from $\mathrm{ZnO}$ nanorod arrays/p-GaN heterostructure devices. Sci Rep 16(6): 25645.

2. Eaton SW, Fu A, Wong AB, Ning CZ, Yang PD (2016) Semiconductor nanowire lasers. Nature Rev Mater 1(6):16028.

3. Sarangi SN (2016) Controllable growth of $\mathrm{ZnO}$ nanorods via electrodeposition technique: Towards UV photo-detection. J Phys D: Appl Phys 49: 355103.

4. Lu MP, Chen CW, Lu MY (2016) Charge-separation kinetics of photoexcited oxygen vacancies in $\mathrm{ZnO}$ nanowire field-effect transistors. Phys Rev Appl 6(5): 054018.
5. Zhu G, Yang R, Wang S, Wang ZL (2010) Flexible high-output nanogenerator based on lateral $\mathrm{ZnO}$ nanowire array. Nano Letters 10 (8): 3151-3155.

6. Fung CM, Lloyd JS, Samavat S, Deganello D, Teng KS (2017) Facile fabrication of electrochemical $\mathrm{ZnO}$ nanowire glucose biosensor using roll to roll printing technique. Sensors and Actuators B 247: 807-813.

7. Lin CH, Chen RS, Lin YK, Wang SB, Chen LC, et al. (2017) Photoconduction properties and anomalous power-dependent quantum efficiency in nonpolar $\mathrm{ZnO}$ epitaxial films grown by chemical vapor deposition. Appl Phys Lett 110(5): 052101.

8. Hussain M, Abbasi MA, Ibupoto ZH, Nur O, Willander M (2014) The improved piezoelectric properties of $\mathrm{ZnO}$ nanorods with oxygen plasma treatment on the single layer graphene coated polymer substrate. Phys Status Solidi A 211(2): 455-459.

9. Yoon IT, Cho HD, Roshchupkin DV, Lee SJ (2018) Enhanced structural and magnetic properties of carbon-supported $\mathrm{ZnO}$ nanorod arrays on (100) Si substrate. Mater Express 8(1): 68-76.

10. Yoon IT, Cho HD, Lee SJ, Roshchupkin DV (2018) Enhanced structural and luminescent properties of carbon-assisted $\mathrm{ZnO}$ nanorod arrays on (100) Si substrate. J Elect Mat 47(8): 4404-4411.

11. Venkatesh PS, Purushothaman V, Muthu SE, Arumugam S, Ramakrishnan $V$, et al. (2012) Role of point defects on the enhancement of room temperature ferromagnetism in $\mathrm{ZnO}$ nanorods. CrystEngComm 14(14): 4713-4718. 